\title{
The SURUGA Gateway: An Advanced CPS Equipping Smart Factory
}

\author{
Guixiang Jin ${ }^{1}$, Akihiro Nakatsuka ${ }^{2}$, and Hiroaki Matsukawa ${ }^{1}$ \\ ${ }^{1}$ School of Science and Technology, Keio University, 3-13-1 Hiyoshi, Kohoku, Yokohama 223-8522, Japan \\ ${ }^{2}$ Fuji Xerox Co. Ltd., 6-1-1 Minatomirai, Nishi-ku, Yokohama, 220-8668, Japan
}

\begin{abstract}
Cyber-physical system (CPS) is one core concept of Industry 4.0. A cyber space has mirror information of physical space and activities in the physical space are synchronized with activities in cyber space. SURUGA Gateway is a CPS, running in a Japanese robot component producer. Innovation process (design) and operation (manufacturing) process is integrated together on the SURUGA Gateway, enabling high quality, short leadtime, low cost, and high flexibility. Various decisions were made in the cyber space, then executed in physical space automatically. Materials input to manufacturing process according to production plan which created by modified ERP (enterprise resource planning) system, fabricated according to optimized routing and scheduling. All NC (numerical control) machines and MC (machining center) were connected and integrated on a centralized platform using Ethercat protocol and interface, and serial electronic signal control for old machines. The KAMISHIBAI (VPS: virtual production system) is embedded in the SURUGA Gateway, providing online training to assembly operators. Innovation process drives operation process, while know-how to improve efficiency and quality in operation process is feedback to the innovation process. The integration of innovation process and operation process, specifically the integration of design, material flow, machine flow and human resource is most successful point of the SURUGA Gateway. SURUGA Gateway successfully reduced leadtime by $80 \%$ and recognized as best practice in Hannover Messe and G20 business conference.
\end{abstract}

Key Words : CPS, mass customization, administration shell, leadtime, management technology, optimization

\section{Introduction}

The concept of platform Industry 4.0 is jointly proposed by BITKOM, VDMA, ZVEI of Germany (BITKOM, VDMA, ZVEI, 2015), and later recognized as Industry 4.0 (Hofmann and Rusch, 2017) and widely diffused to worldwide. From the viewpoint of smart manufacturing, the Industry 4.0 promotes synchronization between cyber space and physical space to maximize production efficiency integrating manufacturing technology, information technology and management technology. Industry 4.0 is a strategy aims to take the initiative in the fourth industrial revolution covering all industry sectors including manufacturing, transportation, energy, agriculture and so on. However, this national strategy does not yet present concrete measures and roadmap for technology development to enterprise real business. The RAMI 4.0 describes some detail frameworks toward future industry development, and therefore many countries launched up numerous pilot projects.

In Japan, SURUGA is selected as one out of ten pilot projects by METI (ministry of economics, trade and industry). We read all reports of ten pilot projects, and selected SURUGA as best practice after ten months investigation including expert interview and factory visits.

Corresponding Author: Hiroaki Matsukawa

School of Science and Technology, Keio University, 3-13-1

Hiyoshi, Kohoku, Yokohama 223-8522, Japan

matsukawa@ae.keio.ac.jp

(Received January 21, 2019)

(Revised March 25, 2019)

(Accepted April 22, 2019)
In this paper, we are going to introduce SURUGA case, specifically focusing on the CPS in SURUGA, which they call SURUGA Gateway.

Regarding synchronization between cyber space and physical space, there is a concept of context awareness to physical space based on RFID (radio frequency identification) technology. RFID technology does provide information of a physical object using standard identification code with microwave data transmission to a computer. However, the RFID technology cannot provide dynamic motion information of a machine or robot.

Recent years, sensing technology has been developed, and it combined with high speed data transmission through the Internet, we can now capable to get and detect dynamically the operation status of a machine and abnormality of an equipment, machine and robot in manufacturing line. RFID technology and sensing technology combined with ICT (information and communication technology) we can trace product flow, we can detect and control machine failure, we can measure efficiency of the manufacturing process. Those data may create Big-data, and the Big-data is analyzed using methodologies of management science and technology which we call the management technology in this paper. From the viewpoint of management technology, mathematical models are important, however data selection and creative ideas to solve problem are more important.

What kind of data should be collected for what purpose is the first coming question when we talk about the 
CPS and Big-data. By analyzing the data using methodologies of management technology, we can forecast product demand more precisely, we can forecast machine failure and determine time of maintenance or machine replacement more properly. Furthermore, those data can also be utilized in the manufacturing scheduling, maintenance, decision-making on spare parts inventory control, and quality assurance.

Since 90 ' s, companies in United States invented many high technologies in the ICT industry, and now so called GAFA (Google, Apple, Facebook, Amazon) applied the technology into business making great success. Cloud computing and data collection, data analysis and utilization, Big-data analysis, in all these latest ICT aspects, GAFA, Microsoft and IBM become world leading companies. In these companies, data not only taken by RFID and sensors, but also taken by mobile terminals, internet, POS (point of sales), electronic commerce, electronic payment and other new ICT tools and equipment.

With respect to manufacturing industry, data collected from production line are used for specific purposes, such as sales and quality control, scheduling, machine maintenance, and etcetera. There are still few cases use data for synchronization between cyber space and physical space in the manufacturing industry. Fewer cases found that integrating innovation process (product design) and operation process (manufacturing).

In other words, although the GAFA, Microsoft, and IBM are front runner, it is not the case that those companies can change manufacturing industry which supports social economy fundamentally. Manufacturing industry requires ICT however they need more mechanical and electronic technologies to invent new innovative products. Integration of ICT and production technology is indispensable, as well as the integration among ICT, production technology and management technology. In other words, we all stand by start line of competition toward next generation of smart factory.

Before describing the synchronization of the cyber space and physical space, we first explain briefly the meaning of cyber space and physical space which we use in this paper. Firstly, cyber space is the space where data is stored and processed, i.e., computers and computer networks. Data and programs were inputted via I/O interface, where sensors and keyboard are frequently used. The internet provides service to connect computer, creating a cyber space, either local cyber space or the global cyber space. Personal computers and smartphones all over the world could connected each other via global/local IP address, DNS (domain name server) and various routers. Distributed database enables the data stored in the hardware which located in far separated place be handled like data stored in one place side by users.

The physical space is the real world where we live in, a factory for example, and is basically consists of product, machine, man, material, and other physical objects. There are various artifacts, such as nature, facilities, buildings, and products, and human beings dig up resources such as ore from nature, make iron, make parts, and produce products in physical space. Since all of these artifacts are based on information on the inherent technology of design, plan, procurement and sales, it is possible to map cyber space to physical space. Needless to say, the ICT is key technology which ties the cyber space and physical space, as well as manufacturing technology and the management technology.

With the advancement of ICT and sensing technology, big amount of data can be automatically obtained and connected. This is one of essential difference in current ICT application compare to the traditional ICT application two decades ago. The national strategies such as Industry 4.0, the Society 5.0, the Advanced Manufacturing Partnership, are launched up based on this new technology that handles Big-data efficiently, transforming data into value added information, knowledge and wisdoms.

The retail Big-data as an outbound logistic data is important for business analysis, and procurement and manufacturing data as an inbound logistic data is also important. Those inbound logistic data may assure product quality, production and operation condition may assure GMP (good manufacturing practice).

The specific technologies such as product design and production process contribute to good design and manufacturing, and the management technology which recognized as a "technology on technology" will contribute to success of the business. The operation process is evaluated by so called iron triangle criterion, quality, cost and due date, and it creates competition power of a manufacturing company. In other words, efficient and good operation, we not only need manufacturing technology, but also need management technology.

The management technology in a systematic approach to control innovation process and operation process so that to maximize efficiency of product design, manufacturing, logistics, sales and after care. Combining and balancing manufacturing technology and management technology became key point for future business competition.

Synchronizing cyber space and physical space occupies one of highest priority for those companies introducing CPS. And this synchronization improves the competitiveness of manufacturing. However, we still have many challenges on this issue, we expect smartness of material flow, machine and human resource utilization. The SURUGA Gateway will provide a concrete case integrating ICT, manufacturing technology and management technology.

The remaining of this paper is organized as follows. In section two, we briefly introduce SURUGA Seiki company, in section three we review literature related to Industry 4.0 and the CPS. In section four, we explain SURUGA Gateway, and in section five we provide discussion and implication. And finally, in section six, we conclude the case study and discuss future perspective.

\section{Suruga Seiki}

Suruga Seiki Co., Ltd. (Shizuoka City, Shizuoka Prefecture, Japan) is a medium-size manufacturer founded in 1964. Their main business consists of (1) develop/produce/sale electric, electronic, communication and industrial equipment and appliances, (2) develop/produce/sale video and optical equipment, (3) de- 
velop/produce/sale parts for industrial machinery. Typical products include, manual stage, automatic stage, controller, light sensor unit, and centering system (see Figure 1). Among them, the manual stage, the automatic stage, and the centering system have the top market share in the world now.

The production can be characterized by built-to-order manufacturing. Most orders have different specification in the SKU (stock keeping unit) level. Suruga Seiki provides over 300,000 SKUs, with mass customization production style. The integration of innovation process and operation process determines their competition power. High precision, high quality and the short leadtime from order to delivery made Suruga Seiki to win market competition and high reputation. For example, industrial robots in manufacturing process need positioning, sometimes need high precision positioning for high quality fabrication. Suruga Seiki can provide stages for those robots to perform one micro level precision positioning. For different robots and machines, Suruga Seiki designs and produces different brand, size, and precision with different positioning methods. This mass customization together with short leadtime makes the innovation process and operation process in the Suruga Seiki complex and difficult.

After CeBIT 2016 in Germany, Society 5.0 strategy is promoted by Japanese government, and many activities toward realizing the strategy are organized with huge amount of budget. Suruga Seiki's products are exactly what helps to achieve maximum precision with harmonized control of operator and robots, by joint utilization of sensing and actuator (see Fig. 2).

[t] Suruga Seiki has 9 subsidiaries including R\& D center, factories and sales department, located in Japan ( 3 ), America ( 1 ), Korea ( 1 ), China (2), Vietnam ( 1 ), and Taiwan (1). Important point is that these subsidiaries are connected by real time base communication network. In other words, all data and problems in subsidiaries are treated in real time base so that operators feel that engineers always stand by their side and help them solve problems whenever they need.

There are many management information systems supporting planning, scheduling, merchandizing, manufacturing control, design support, however those functions are supported separately and data between the separate systems were communicated with analog style such as phone call, facsimiles, keyboard input and so on. Even an advanced ERP (enterprise resource planning) and Web-EDI (web electronic data interchange) service do not provide such kind supports all data and information connected each other due to the different standard, business style and culture. A real time base network must use unique code for each product and all local application program could automatically treat data from other department or functional divisions.

\section{Literature Review}

Industry 4.0 strongly emphasizes standardization, and this is one important difference between the Industry 4.0 and the Society 5.0 [24][23]. Society 5.0 emphasizes key technologies, ICT platforms, social infrastructure, welfare and happiness, however one cannot find business oriented or management technology issues including initiative on international standardization. Made in China 2025 emphasizes technologies too, and we found weak description on synergy effect of technologies and standardization in the strategy [16], even in some Chinese documents directly declares they follow standards of Industry 4.0.

Lukac [17] defined Industry 4.0 as the fourth industrial revolution characterizing it with cyber physical systems (CPS) production, based on heterogeneous data and knowledge integration. Luka defined that the first industrial revolution started from end of the 18th century with representative technology of water and steam power; the second industrial revolution started from early 20th century with representative technology electric power; the third industrial revolution started from early 1970s with representative technology automation and Internet technology.

We can also find many papers discussing industrial revolution, however we did not find papers discussing industrial revolution as a revolution involving society and system engineering. Indeed, key technology is necessary condition for industrial revolution, however not sufficient condition. We believe that those key technologies must be embedded into a system, such as a factory with steam engine power, factory with electronic power. And we further need that the improved business model including manufacturing process, financial system, sales management, incentive design, optimal control of business process, and so on. In other words, we need new management technology for those new technologies.

From the viewpoint of key technology, we could find many papers on Industry 4.0.[26] proposed five major features of the Industry 4.0, i.e., (1) digitization, optimization, and customization of production; (2) automation and adaptation; (3) human machine interaction (HMI); (4) value-added services and businesses, (5) automatic data exchange and communication. Recently, we organized interview to manufacturing companies, research institutes, and government of Germany and Japan. Through the interview, we found that "digital transformation (DX)", "CPS", "connected", and "cost efficiency" are four major concerns with respect to the Industry 4.0 in Germany and Japan. Surprisingly, automation was not top priority for most manufacturing companies. Instead, supplier management and demand matching which belong to management technology or supply chain management have had higher priority than automation.

Up to 2017, there were more than 100 academic papers have been published on the research topic Industry 4.0 [15]. Lu (2017) conducted a systematic literature review using the keyword, "Industry 4.0", and classified 88 papers into five categories, i.e., (1) Concept and perspectives of Industry 4.0 (18 papers), (2) CPS-based Industry 4.0 (12 papers), (3) Interoperability of Industry 4.0 (11 papers), (4) Key technologies of Industry 4.0 (20 papers), (5) Applications of Industry 4.0 (27 papers). Most of these papers provided constructive proposals, from the viewpoint of standalone technology. However, these proposals not yet implemented in the practice. 


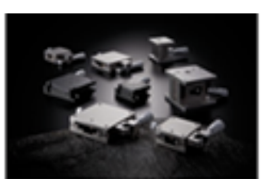

Manual stage

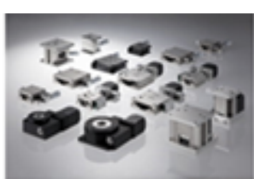

Automatic stage

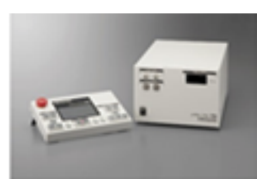

Control unit

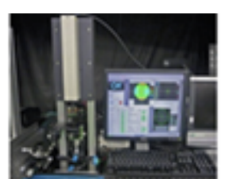

Optic sensing unit

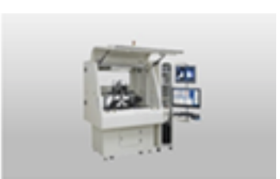

Centering system

Fig. 1 Typical product group of Suruga Seiki Co., Ltd.

\section{Equipment of Process Automation}

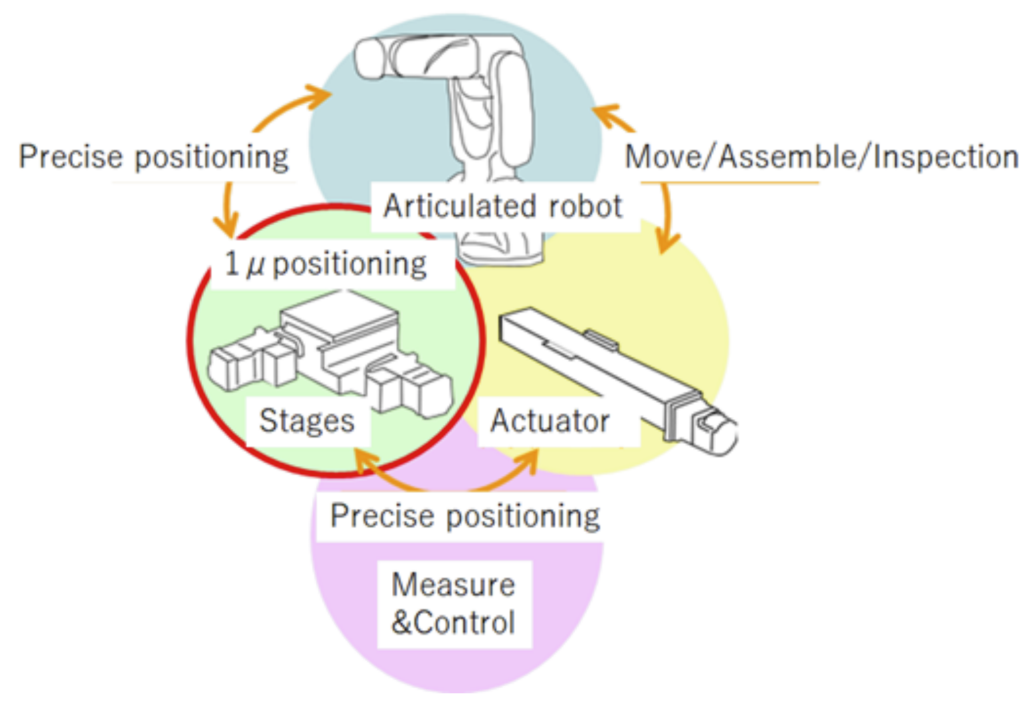

Fig. 2 Supporting multi-jointed robots with sensing and actuators

\subsection{CPS}

Research papers are expecting that the CPS can provide promising solutions to transform the operation and role of many existing industrial systems [8][20][38]. ICT is indispensable key technologies in the CPS construction. One example of the CPS concept is the architecture proposed by [18].

Concerning ICT infrastructures, we also found many papers which arms to contribute CPS building [5][13][11][18] [21] [22][33]. The CPS can be defined as "the convergence of the physical and digital worlds by establishing global networks for business that incorporate their machinery, warehousing systems and production facilities" from the ICT infrastructure viewpoint (Cyber space, [33]. The CPS can also be defined as "systems of collaborating computational entities which are in intensive connection with the surrounding physical world and its on-going processes, providing and using, at the same time, data-accessing and data-processing services available on the Internet" [21]. Data can be collected using sensors, edge computers, and operators, with gesture and motion capture and/or but- tons.

Collected data are analyzed for preventive maintenance, production process control, rescheduling and so on [19]. Important point of the CPS is synchronization. Data collected from field will be contained into the cyber space, i.e., computer system, and analyzed according to some predetermined automatic logic and/or interaction between computer and decision maker. Once decision is made either automatically or manually, it will feed beck to physical space to control action of machine and operators. This kind of synchronization plays important roles in improving the overall industrial performance [9]. CPS is a wide concept to increase productivity, workforce performance, and assure higher quality with lower costs [30]. Different industrial sector, and different manufacturing company may have different features of the CPS, so that to fit their customers, machines, operators and suppliers.

\subsection{Application of CPS}

Jazdi[11] presents an application of CPS. In their research, work processes and development methods in CPS are re- 
defined. Some CPS requires coordination between activity and activity in manufacturing procedures, while some dynamic optimization methods could be applied analyzing data collected from production line [9]. Under the idea of the dynamic optimization methods, Ivanov proposed a service-oriented dynamic optimization model, i.e., dynamic scheduling and collaboration.

There are several practical ideas which support CPS application in the innovation process and operation process. Shafiq [33] proposed three application ideas, i.e., (1) Virtual Engineering Objects (VEO), (2) Virtual Engineering Process (VEP), and (3) Virtual Engineering Factory (VEF). VEO deal with knowledge transition through data mining finding experience of engineering artifacts. The experience could be used design and manufacturing so that to improve efficiency [6][12][31][33]. VEO can increase flexibility of a CPS, enabling reconfiguring in an operation process. Accumulated knowledge can improve design efficiency, as well as manufacturing efficiency [26]. VEP can perform a virtual manufacturing, collect operation-required information, while the VEF represents an experience-based knowledge in an engineering factory [33].

For extended research and application on the CPS, we have two major concerns. The first one is how to implement the concept of CPS to real business integrating various resources and processes, such as material flow, machines, order processing and so on. The second concern is how to improve CPS continuously when market environment changes. The advances in ICT such as $5 \mathrm{G}$, sensor network will provide wider rooms to improve for the CPS, and new ideas become easier to be implemented in a manufacturing company [37]. Distributed remote applications could be another possible future application concerning advanced CPS [11]. As one of extended CPS, Monostori et al.[21] proposed cyber physical production systems (CPPS), which more emphasize computational effort, data-related procedures, automation, and ICT. The CPPS involves not only machines and products but also operators, and emphasize integration of edge computing, communication, networking, data analysis, and higher capability on improving key performance indicator (KPI) in particular, quality, cost, and delivery (QCD) and scalability $[2][19]$.

Production process model (PPM), the information exchange model (IEM), and the plant information model (PIM) which support efficient CPPS are proposed by Perez et al.[28]. An application of the CPPS is in healthcare is reported [29]. In the application, the system tracks patients via mobile application, sensor, and surveillance in apartment so that to provide timely service.

One another case is to develop a network of VEO. The VEO provides application of engineering artifacts from simple stand-alone artifacts to complex multitask machines $[6][26][33]$. The VEO can add, store, improve, and share knowledge [33].

\subsection{Smart factory}

Smart factory is one key components in Industry 4.0. Main characteristics of the smart factory includes, flexibility, data driven, connected, and autonomous [29][1]. Accordingly, one precondition is that machines are equipped with data collection sensors, reacting emergency quickly with edge computing, and automation. Intelligent is another keyword for smart factory, which enables finding and solving problems that occurs in manufacturing process, and control material flow efficiently. The smart factory is regarded as a kind of intelligent factories which is the major goals of Industry 4.0 [32]. Multi-agents simulation used in manufacturers' resource planning [3], and value-added integration either horizontally or vertically or mixed style in the manufacturing process becomes one trend [34][35]. Value creation modules from the material procurement to the logistics expanded and implemented horizontally, whereas the vertical procedure integrates product, equipment, and operators. The Industry 4.0 drives manufacturing in two directions, i.e., (1) application-pull and (2) technology-push [19]. Application-pull is easier to be introduced which has background of fierce market competition and promising return on investment. Energy conservation measures (ECM) is one another concern in smart factory [25]. Using high frequency energy meter, a model to estimates the risks, measure and verifying energy conversation is proposed [25]. Virtual manufacturing which is similar to the simulation in a PLM (product lifecycle management) system is proposed consists of three entities, i.e., virtual engineering objects, virtual engineering processes, and virtual engineering factories. Three entities integrated and construct one relatively complete smart factory operation system.

The literature review shows that smart factory requires synchronization of material flow and operation flow [7] $[9][10][27][30]$. Cloud manufacturing is one new concept which save cost of upgrading of manufacturing execution system, management information system and so on [36].

Through literature review, we found that many concepts are proposed toward improvement of efficiency of innovation process and operation process, and stand-alone technologies has been developed to improve productivity without management technology which enable the technology became feasible solution in business. In this paper, we introduce one real case, the SURUGA Gateway, a special CPS for mechanical component producer.

\section{SURUGA Gateway (Suruga Seiki's CPS)}

SURUGA Seiki co. Ltd. is selected one of ten pilot projects of Industry 4.0 by METI in Japan. Their achievement improved KPI of the company, and also be selected one of best practice in Japan. In Hanover Messe 2018, SURUGA Gateway received wide attention and highly evaluated. SURUGA Gateway consists of four parts: (1) Front-loading system, (2) CAD/CAM/VPS, (3) scheduler $\&$ MES, and (4) Suruga version administration shell.

\subsection{Front-loading system}

Firstly, the Front-loading system built with parametric stage design system, letting customer determine product specification with selecting predetermined table size strokes, allocate tap hole numbers and positions, then the system make cost estimations based on existing models, 


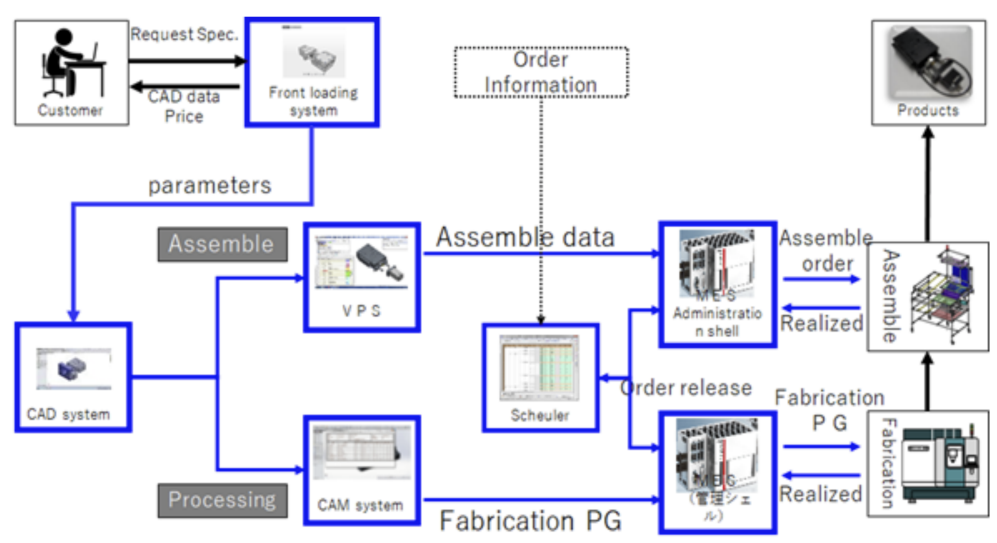

Fig. 3 Overview of SURUGA Gateway

and create native 3D data and give immediate feedback of design, the tap hole, spot facing hole type, position, and required motor type etc. The concept of Front-loading includes dividing the design stage in several phases and carrying out concurrent engineering (CE), and early involvement of other division including manufacturing, procurement and so on. Suruga Seiki's Front-loading is characterized by integrating operations, design, and manufacturing to quickly answer due data and feedback price estimation to customers' inquiries, not simply apply traditional concept of Front-loading. Also, when an order comes in, the Front-loading system swiftly generates a parameter for the backbone system, sends it to the manufacturing department and reduces the manufacturing leadtime. In other words, using a new front-loading method, not only has the leadtime of the conventional design and manufacturing been reduced together with the refactoring, but also design, manufacturing, and marketing unified on the SURUGA Gateway so that to promptly respond to the customers' orders.

\section{$4.2 \mathrm{CAD} / \mathrm{CAM} / \mathrm{VPS}$}

In $\mathrm{CAD} / \mathrm{CAM} / \mathrm{VPS}, \mathrm{CAD}$ data is automatically generated using the parameters offered and generated by the front-loading system. Same time, E-BOM (engineering bill of material) and 2D design file are generated automatically for manufacturing. Using CAM (computer added manufacturing) system, Suruga constructed skill-less manufacturing environment through master data creation, maintenance and utilization. In other words, an operator does not need input fabrication program each time considering materials, machines, tools, temperature, etc., and the automatically generated fabrication program will assure high quality fabrication in which current condition of the machine and shop are considered in the program generation. The operator prepares tools based on the output sheet of the CAM system and associated fabrication program. The VPS (virtual production system) creates data such as assemble sequence, method, and associated tools, then demonstrate it on monitor front of operators who need it. VPS acts as an e-learning system in manufacturing line and surely it is powerful tool to support skill-less assembly and quality assurance.

\subsection{Scheduler and MES}

MES is a software releasing production order in the process industry such as petroleum and chemical industries. Since the logic of production in processing industries and assembly industries are similar in terms of production flow, the MES are popularly used in the assembly industry or discrete type production. MES is different from production management software such as MIS (management information system) or MRP (material requirement planning). MES is adjunctive software connecting MPS (master production schedule), MRP and release order to machines, collect data and evaluate performance.

Suruga Seiki's MES first receives production related information such as demand from the production management system. Demand information flow into IPC (innovative production system, original core of Suruga Seiki's MES), and the IPC creates all consequent operation data, including processing sequence (schedule), fabrication program for each NC. Scheduling software provides a visual check function through Gantt chart and firm fixed schedule finalized by a person who responsible for performance of the scheduling. Although the scheduling is not generated based on a mathematical model, it works well generating feasible solution quickly and improved through interactive tuning by a skilled planner.

\subsection{Suruga administration shell}

The role of Suruga administration shell (SAS) is to connect the machines (physical space) and management system (cyber space). Machines and equipment are located in lower layer in the SAS, while production schedule is located in higher layer. The SAS consists of resource manager, protocol compartment, MES, manifest, and some other specialized functions. The host system is a business 
management system supported by ERP. In order to meet business demands, the SAS first receive production information associated with product parameters and general programs, then hands them over to MES, creates production scheduling, and converts the general program into a specialized processing program to each NC machine according to machine assignment and operator assignment that determined by production schedule. The special processing program is converted to a processing program capable of controlling machines of different vendors and different protocol compaction, then actual processing is performed based on MES information. When an operation is completed at one machine or process, parts will be carried to the next process, where they are processed according to operation commands created by the administration shell of next process.

Since the detail definition of the administration shell are different in each company and it depends on product, we cannot say that the Suruga SAS is best. The only thing we can say is that the SAS really works and supports Suruga' s quality and due data. There are also many restrictions on the administration shell, and this might be a good reason that each company have different administration shells which depends on the product. However, we may have some common structure and modules, no need to start everything from scratch, and it will be an efficient way to create administration shell based on a common standard platform.

\subsection{The VPS (Suruga's KAMISHIBAI)}

An assembling sequence and operation method are required when assembling products. Traditionally, an assembly operator is trained to perform correct operations by other assembly operators who have high skill and knowhow on the assembly operation. After off-line training, they will be assigned to assembly line. One operator should understand the structure of the products and the process of his own tasks, making him able to operate efficiently within few days training or OJE (on the job education). Suppose that one operator performs all assembly operation for one product, i.e., one pure cell production, it would take a long time and cost for training. In mass customization manufacturing with as many as 300,000 SKU, like Suruga Seiki, the efficiency of this training time is crucially important for assure quality and due date. For establishing efficient way to ensure high quality operation of the assembly operator, Suruga Seiki created the assembly operation manual automatically using data from CAD/CAM system. The assembly process displayed to the monitor which set at front of an operator, and the operator can perform correctly checking parts, tools and assembly orders step by step shown on the monitor. Operators will learn operation sequence and method which supported by lamp lighting, telling the operator to pick up correct tools, take correct parts, and assemble it correctly watching the monitor, which Suruga calls the KAMISHIBAI. If the operator picks up wrong tool or wrong part, the VPS alarms the operator check and take correct tool or parts. This is quite similar to so called POKAYOKE in Toyota Production System.

\section{Process of Construction and Major Func- tions}

Bottom up style improvement is recognized as a typical Japanese culture in manufacturing companies. One factory is divided into several departments, and each department sets the goal for one year in accordance with the goal of the factory top manager. Division managers have power and responsibility for all performance on quality and due date. One can find similar facts in street across in Japan. Peoples never walk cross when red signal lighting, and when they walk through congested cross, Shibuya scramble across in Tokyo for example, peoples watching and cooperate each other so that to avoid collision. A Japanese manufacturing company have a set of rules and working manner, and managers and operators keep the rules strictly and behaves supportively for those issues that company rule does not cover. Moreover, managers and operators have a mind which is called problem finding that similar to the quotation "a good question is more valuable than a good idea".

Synchronization among different departments is supported by a Japanese culture which called "SURIAWASE". When operators find problem, even in the process of problem finding, they communicate each other, stimulate each other, in or out of working times. This process is called "integration process" from management viewpoint. This may bring much benefits for a factory to create seamless material flow without having complicated long rules set. In the sense of an autonomous decentralized system, we can say that Japan's bottom-up management style is a good system that responds swiftly to environment changes.

However, when we consider synchronization of cyber and physical space, such bottom-up management style does not work well. This is because coordination between organizations in cyberspace cannot be supported by so called “SURIAWASE” or “integration”. In cyber space, all instances must be connected and processed according to a set of rules strictly. In other words, the coordination in the CPS is a new technology and a new system that enables optimization over the factory operation, therefore we need a new approach which fit to the CPS.

Suruga Seiki, combined bottom-up and top-down approach in their practice of SURUGA Gateway construction. Top-down approach integrates entire manufacturing system including design, fabrication and assembly, transfer automatically generated fabrication programs to machines, use CAD data in assembly operation (VPS), receive the ERP data with EDI and input it to the scheduler before issuing manufacturing orders to the manufacturing process, and so on. Suruga Seiki conducts these design and manufacturing activities in physically separated regions and countries, places the connections in the CPS that makes the products from design information available everywhere inside the cyber space, the integrated manufacturing platform. This platform enables the bottom-up approach works too. Although the design and manufacturing operations are located in physically separated locations, they are seamlessly connected within the integrated 


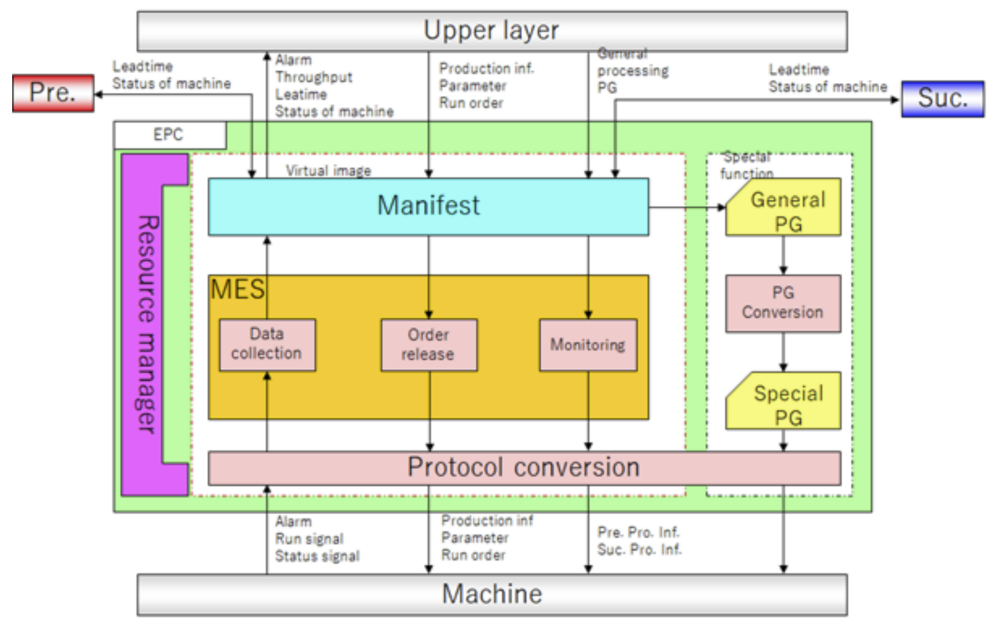

Fig. 4 Administration shell of the SURUGA Gateway

cyber space, and operators use the platform as a tool of efficient communication.

Integration of top-down and bottom-up approach is correct way for the CPS construction, however for different product, different culture, different process we need different process of integration in practice.

\subsection{Design and development process}

SURUGA Gateway development motivated and supported by Japanese government METI (ministry of economics, trade and industry). For pilot project, Suruga developed the system under guidance of the Industry 4.0. They clarified the position of the CPS in RAMI 4.0 first, then proceed to detail design of the structure and functions. Many challenges and tasks carried out and finally Suruga successfully implemented the system into their innovation process and operation process, visualizing whole manufacturing process including marketing design, fabrication, assembly and delivery. They were benefited by the visualization finding problem quickly therefore solve problem efficiently using collective intelligence on the platform.

There are four steps to develop the CPS system. The first step is the positioning, the second step is to define administration shell, the third step is to design hardware and software, and the last step is to build the complete CPS system.

In positioning step, Suruga chosen the production process on the axis of lifecycle \& value stream of the first axis (see RAMI 4.0). Machine tool and assembly equipment on the 2nd hierarchy levels axis. And in the third axis layer, Suruga chosen information. Administration shell is defined by five layers, i.e., (1) Object/Entity, (2) Model/Instance, (3) Communication Capability, (4) Virtual Representation (Data), and (5) Function. All objects related to design and manufacturing which can be classified into four functions:

(1) Communication skills: a passive object communication ability,
(2) The Manifesto: virtual image data of each target,

(3) The resource manager: operation ability, such as time management,

(4) The professional characteristic of the object function: function beyond data processing.

As mentioned above, the object of the administration shell consists of physical machines and control software, and operators use it as a tool in the manufacturing process.

In design, in order to respond to different work load in the office floor and the shop floor, the entire system is covered by mechanism/measuring equipment (bottom level, edge computing), equipment / control equipment (middle level, machine tool / equipment), and upper management system (upper level, system integration), respectively. Three level management system enables smart control and manufacturing. For more detail, the bottom level component corresponds with the mix of hardware and software making full use of standard I/O, serial, field bus etc., so that to ensure equipment works properly. In middle level, administration shell integrates and utilizes FA (factory automation) technology, MES, scheduler, and $\mathrm{CAD} / \mathrm{CAM} / \mathrm{VPS}$.

Suruga evaluated fitness of their CPS system based on the "component definition of RAMI 4.0" and published the results in Hannover Messe, and those organizations original proposer of Industry 4.0, the BITKOM, VDMA, and ZVEI highly evaluated SURUGA Gateway as the best practice in the world. SURUGA Gateway also presented in the G20 conference and received high evaluation.

\subsection{Suruga Supply Chain (SSC)}

Supply chain management (SCM) mainly focus on products and associated information flow and monetary flow. However, there are two another important resources to support product flow, i.e., machine and operator. From the viewpoint of manufacturing, the machine and operator are crucial to organize efficient product flow in factory. We 
assign machine and operators to perform jobs to produce product, sometimes changing layout of machines. This means that machine and operators can also be seen as two important flow inside a factory to form internal logistics. In other words, when we consider a supply chain, either internal or external, we should consider not only the product flow, but also machine flow including vehicle, and the operators flow including part time workers. An integrated management of these flows is required for new generation of SCM and Sruga did it. Machine manufacturer is vendor, which is not included in the traditional supply chain management, however machine flow including investment and layout change are quite much important or have influence to product flow in a supply chain.

Suruga Seiki is striving for vertical integration of the supply chain (design, procurement, manufacturing, marketing), and the SURUGA Gateway provided an information platform to realize it. Interestingly, Suruga introduced new concept of supply chain management involving machine vendors. Machines purchased from different vendors are integrated on SURUGA Gateway, fabrication programs for different machines from different vendors and different year were automatically created by the SURUGA Gateway after the customer's order was firmly fixed. Connecting machines and assign jobs, providing manufacturing schedule and fabrication programs, different routing was selected maximizing system-wide manufacturing efficiency. And this kind dynamic routing was included in the SSC management.

If a company relies on operator's skill, manually controls and operates either old machine or advanced NC machines, then operator training becomes a problem. Operators have to learn many control languages for each machine which produced by different vendor. It may take time to understand and remember control command and fix problems which occur during fabrication. Now, many vendors are introducing so called platform providing standard fabrication programs for different machines. However, it covers only their own machines, therefore, a user company such as Suruga have to spend time and cost to learn different control languages purchased from different vendors. Essentially, those platforms more likely be introduced for the purpose of marketing, forming up user's loyalty. Suruga' s SSC could be one possible solution and direction so that all machines could be controlled by one platform without spending time to read thousand pages of manuals.

Suruga Seiki proposed an open communication data generation technology that easily generates machining programs, connecting and routing dynamically the machines purchased from different vendors, old and new machines. It will increase user's manufacturing efficiency drastically with the proposed platform, enabling a quick generation of processing program using product CAD data. This is one of the challenges for small and medium-sized companies that have a significant shortage of high skill operators in Japan. Vendors should not have to stick on the local control language, they should consider the efficiency of their customer' s convenience and efficiency, so that the users use their products efficiently and comfortably. The vendors, even if they are competitors, they should cooperate each other to build a new and common platform. This is an important issue for national industry and competition power.

\subsection{Optimal production routing and parameter setting}

Machines such as lathering, flywheel, balling, and polishing are used for parts processing. These machines are now grouped and connected, and parts are processed using this grouped and connected.

The quality requirement is severe in Suruga, because of the precision requested by customers. Although it is possible to inspect $100 \%$ parts one by one and remove defective products, however more importantly, prevent defective parts from occurring during the manufacturing process may increase efficiency of production and decrease cost. Building quality in the manufacturing process is also an ironclad rule of quality control, which is the strength of Japanese manufacturing industry. Good materials, tools, equipment, and processing method are important to build good quality. Fredrick Winslow Taylor, the father of scientific management, tackling lathering problem spending 20 years and 200,000USD costs, find out best way to determine two operation variables under 13 different parameters. In order to standardize the lathering machine operation, he conducted research for all several million combinations of various processing conditions, such as material, strength, and feeding speed of cutlery (cutting thickness), he finally invented a sliding calculation ruler, which enables a new operator can determine an optimal machining method (turning speed, feeding speed of cutlery). In Suruga, optimum processing method, including routing and fabrication program, with 20 parameters (independent variables), were created within an hour, based on analysis of 400 thousand records using deep learning. More importantly, it improves evolutionally with increasing new data collected from machine side and final product side. It can be considered that the Taylor's sliding calculation ruler improved every day.

There are three technologies for manufacturing competition, i.e., product technology, manufacturing technology, and management technology. In many situations, competitiveness is determined by manufacturing technology and management technology. In many situations, materials, equipment, and product specifications are open and homogeneous. The product technology is mostly public information due to the open patents, and the imitation can be easily carried out decomposing and analyzing the product. However, a high precision product is not the case, because of the hidden know-how and manufacturing technology which mostly not exposed to market with patent. Since it is not possible to protect the optimal product processing procedure by patent, we should carefully consider the release of manufacturing technology and management technology. The platform business has merit in the situation if a synergy effect can be obtained or the resource sharing is possible, and there is a feature with the platform where all players can increase sales and increase customer's royalty. The important point for the manufacturing industry is to construct strong supply chain, including procure- 
ment and distribution, to build secure value chain. The platform for this purpose is necessary and we consider it is beneficial for a manufacturing company. Japanese assembly manufacturer should not set so called "SURIAWASE" (Fujimoto and Kim, 2009, Inoue M., Mogi R., Nahm YE., Ishikawa H., 2011) as belief, manmade complicated design, with high cost throughout product lifecycle. Suruga case tells us that the right way is to promote modularity and standardization as much as possible, so that to reduce cost in matured technology, while keep benefit from the SURIAWASE for advanced new manufacturing technology and management technology. Suruga case also tells us a fact that the CPS may replace some traditional methodology such as experimental design. Big-data which generated from the CPS will feed back value added information to decision-making using management technology. Big-data and computation power may replace large part of traditional statistical method which used in product design, quality control, and manufacturing routing and fabrication program.

\subsection{Comprehensive production}

The important features of SURUGA Gateway its capability to produce and deliver over 300,000 SKUs within 7-14 days. As the best knowledge of authors, this kind of mass customization in business to business style manufacturing, Suruga provides one best practice in the world.

Although one can change the design by using traditional CAD, without automation and integral management of the CAD, CAM and VPS, it is not possible to realize short time to market with high quality. The SURUGA Gateway enables integral management of design, procurement, manufacturing, and marketing, their practice shows us it is possible that we can build a comprehensive production system. The Suruga' s comprehensive production system is supported by which we call the "open communication and data generalization technology".

The Suruga's comprehensive manufacturing system works as follows. First, Suruga prepares categorized platform product which generated by historical CAD data. Second, the customer input product name and demanding parameters for their customized requirement. Third, the SURUGA Gateway feedbacks first draft production design and price estimation. Then, customer changes parameters to create their imagined product and finally price is determined with few round negotiations.

As soon as Suruga get firm fixed order from customer, product design is completed and firmly fixed. Production scheduling and routing is generated assigning machines and associated fabrication program (PG) immediately. Production order is released according to the production scheduling, and operators can see pop up fabrication order on the monitor. Interestingly, an operator in a production shop located in Shanghai (China) can respond the production order generated in Shizuoka (Japan) within 5 seconds. Neither phone call, nor email. In assembly, the VPS generates assembly animation video and required tools for each operator, and the operator assembles parts observing and confirming the animation video. Fool proof system prevents the operator from using wrong tool and took up wrong parts. After several round assembly, with the learning curve effect, assembly efficiency increases significantly.

The master manufacturing plan is generated using orders come from customers, and transmitted to factories using EDI communication, and converted into a schedule that can be executed in each production shop. An advanced scheduler creates best first optimal schedule and routing in which the schedule can be changed manually, and all related operation schedule are automatically changed according to constraint propagation. After quality check, those assembled products will proceed to final packaging process. Various bottom-up improvement activities were performed and increased efficiency. In this paper we present several examples of bottom-up improvement in next section.

\section{Bottom up approach: the KAIZEN}

The SURUGA Gateway is built up integrating top down and bottom up approach. Suruga seiki also increased operation efficiency as much as possible by using traditional bottom up approach. One typical example is packaging material and operation.

In order to prevent the damage of the product by the vibration during transportation, Suruga operators designed one special packaging box for their product. A stretchable plastic sheet (environmentally friend material) is sealed on a thick packaging plain material first, bending the plain operator insert product (Stage) into the gap between plastic sheet and plain material. The product is fixed on it after relaxing the bended plain. Two side of the plain inversely bended to fit the size of box, then putted into the box, hanging the Stage on the center of the box. After close the box, it become a high specification packaging box with cushion function. The cost of the packaging is low and environmentally friend. If drops the box from meter height top, then corner of the box is crushed, however the part is protected perfectly.

One another case of improvement is the screw tightening operation. One single operator or an operator's team can get technological, managemental and financial support from division manager, then launch up KAIZEN project. For the screw tightening operation, operators invented one automatic bolt supply slide where randomly loaded bolts will change directions and correctly line up when it passes through the bolt feed machine, and four bolts will be inserted into four holes same time and the screw tightening machine with precise torque come to tighten four bolts quickly. This simple machine greatly increased efficiency of the screw tightening operation and quality releasing operators from boring hard work.

One interesting improvement activity is that Suruga engaged into improvement activities in their customer companies. Suruga marketing engineer knows how their product could improve performance in their customer' $\mathrm{s}$ business, and they will provide advice or change product design for their customer company, so that to increase their service level and increase their customer's loyalty. For example, Suruga Seiki has contributed to simplified robot and production line in a customer company by adapting 
modified automatic Stage, contributing to improvement in accuracy and high yield of the smart panel bonding process. In this way, rather than promote product sales, their proposal on problem solving in customer company are more welcome and even earns more profit in long term viewpoint. From Suruga case, we can conclude that an improvement should not only be internal, but also external. Suppliers know main characteristic of their parts well, therefore may come up with new ideas when they see where the parts are used.

\section{Conclusion}

In this paper, we conducted a case study of CPS, the SURUGA Gateway. Suruga built a CPS to improve QCD, integrating innovation process and operation process. Suruga case shows that mass customization is capable for such small product, high quality and high precision. Suruga reduced leadtime drastically through the SURUGA Gateway, keeping mass customization. Quick estimation, quick program generation for machines, quick production routing, shorter training time for assembly operator are all excellent practice to learn.

Suruga Seiki's case shows that not only manufacturing technology but also management technology is important to build a new CPS. Mathematics used in tactical decision making in Suruga currently, optimal routing and scheduling for example, however mathematical model can also support better decision making on the strategic problems. Product technology, production technology and management technology must be integrated together so that to maximize efficiency of a business reengineering.

Suruga Seiki are creating and utilizing data such as product image recognition record and associated manufacturing routing and operation conditions, such as material, torque, speed, temperature and so on. Data is automatically converted to value added information which can help proper decision making for managers and operators.

One remaining problem is production scheduling. Although we can generate theorical optimal production scheduling, uncertainty of environment will destroy the optimality. The important thing is to improve scheduling methodology and associated software which may support decision maker to generate a new feasible schedule quickly.

\section{Acknowledgements}

We thanks to all supports and comments from Suruga Seiki. All figures are generated by the Suruga smart factory team members. We would not complete this paper without their supports.

\section{References}

[1] Anna Rymaszewska, Petri Helo and Angappa Gunasekaran (2017). IoT powered servitization of manufacturing - an exploratory case study, International Journal of Production Economics, 192, 92-105.

[2] Albers, A., B. Gladysz, T. Pinner, V. Butenko, and Sturmlinger, T. (2016). Procedure for defining the system of objectives in the initial phase of an Industry 4.0 project focusing on intelligent quality control systems, Procedia CIRP, 52, 262-267.

[3] Adeyeri, M.K., K. Mpofu, and Olukorede, T.A. (2015). Integration of agent technology into manufacturing enter- prise: a review and platform for Industry 4.0. International Conference on Industrial Engineering and Operations Management (IEOM), IEEE, 2015, 1-10.

[4] BITKOM, VDMA, ZVEI (2015). Survey report on platform Industry 4.0: the Industry 4.0 implementation strategy, Berlin. (In Japanese, translated by JETRO).

[5] Bagheri, B., S. Yang, H.A. Kao, and Lee, J. (2015). Cyberphysical systems architecture for self-aware machines in Industry 4.0 environment, IFAC-Papers On Line, 48, 16221627.

[6] Brettel, M., N. Friederichsen, M. Keller, and Rosenberg, M. (2014). How virtualization, decentralization and network building change the manufacturing landscape: an Industry 4.0 perspective, International Journal of Mechanical, Aerospace, Industrial, Mechatronic and Manufacturing Engineering, 8 (1), 37-44.

[7] Chen, Z. and Xing, M. (2015). Upgrading of textile manufacturing based on Industry 4.0. 5th International Conference on Advanced Design and Manufacturing Engineering, Atlantis Press.

[8] Gurdur, G., J. El-Khoury, T. Seceleanu and Lednicki, L. (2016). Making interoperability visible: data visualization of cyber-physical systems development tool chains. Journal of Industrial Information Integratioin,4, 26-34.

[9] Ivanov, D., A. Dolgui, B. Sokolov, F. Werner and Ivanova, M. (2016). A dynamic model and an algorithm for shortterm supply chain scheduling in the smart factory Industry 4.0, International Journal of Production Research, 54 (2), 386-402.

[10] Ivanov, D., B. Sokolov, and Ivanova, D. (2016). Schedule coordination in cyber-physical supply networks Industry 4.0, IFAC Papers On Line, 49 (12), 839-844.

[11] Jazdi, N. (2014). Cyber physical systems in the context of Industry 4.0. IEEE International Conference on Automation: Quality and Testing, Robotics, IEEE, 2014, 1-4.

[12] Kobara, K. (2016). Cyber physical security for industrial control systems and IoT. IEICE Transactions on Information and Systems, 99 (4), 787-795.

[13] Harrison, R., D. Vera, and Ahmad, B. (2016). Engineering methods and tools for cyber-physical automation systems. Proceedings of the IEEE, 104 (5), 973-985.

[14] Hofmann, E. and Rusch, M. (2017). Industry 4.0 and the current status as well as future prospects on logistics. Computers in Industry, 89, 23-34.

[15] Lu, Y. (2017). Industry 4.0: A survey on technologies, applications and open research issues, Journal of Industrial Information Integration, 6, 1-10.

[16] Li, D. Q. (2014). Talk on Made in China 2025 strategy. Science and Technology Daily News.

[17] Lukac , D. (2015). The fourth ICT-based industrial revolution "Industry 4.0"; HMI and the case of CAE/CAD innovation with EPLAN, 23rd Telecommunications Forum Telfor (TELFOR), IEEE, 835-838.

[18] Lee, J., B. Bagheri, and Kao, H.A. (2015). A cyberphysical systems architecture for Industry 4.0-based manufacturing systems, Manufacturing Letters, 3, 18-23.

[19] Lasi, H., P. Fettke, H.G. Kemper, T. Feld, and Hoffmann, T. M. (2014). Industry 4.0, Business Information and Systems Engineering. 6 (4), 239.

[20] Mao, J., Q. Zhou, M. Sarmiento, et al. (2016). A hybrid reader transceiver design for industrial Internet of things, Journal of Industrial Information Integration, 2,19-29.

[21] Monostori, L., B. Kadar, T. Bauernhansl, S. Kondoh, S. Kumara, G. Reinhart, and Ueda, K. (2016). Cyberphysical systems in manufacturing, CIRP Annals Manufacturing Technology, 65 (2), 621-641.

[22] Mosterman, P.J., and Zander, J. (2016). Industry 4.0 as a 
cyber-physical system study, Software and System Modeling, 15 (1) (2016) 17-29.

[23] Maeda, A. (2018). Toward “Society5.0" from a perspective of technology-oriented innovation, Oukan, 12(1), 28-32(in Japanese).

[24] Nananishi, H. (2016). Society 5.0 and 5th national strategy of science and technology. CRDS Symposium, Keynote speech, Organized by Japan Science and Technology, Tokyo, Japan.

[25] Oses, N., A. Legarretaetxebarria, M. Quartulli, I. Garcia and Serrano, M. (2016). Uncertainty reduction in measuring and verification of energy savings by statistical learning in manufacturing environments. International Journal on Interactive Design and Manufacturing, 10 (3), 291-299.

[26] Posada, J., C. Toro, I. Barandiaran, D. Oyarzun, D. Stricker, R. de Amicis, and Vallarino, I. (2015). Visual computing as a key enabling technology for Industrie 4.0 and industrial internet. IEEE Computer Graphics Applications, 35 (2), 26-40.

[27] Pisching, M.A., F. Jnnqueira, D.J. Santos Filho, and Miyagi, P.E. (2015). Service composition in the cloudbased manufacturing focused on the Industry 4.0. Doctoral Conference on Computing, Electrical and Industrial Systems, Springer International Publishing, 65-72.

[28] Perez, F., E. Irisarri, D. Orive, M. Marcos, and Estevez, E. (2015). A CPPS Architecture approach for Industry 4.0, IEEE 20th Conference on Emerging Technologies \& Factory Automation (ETFA), 1-4.

[29] Roblek, V., M. Meko and Krape, A. (2016). A complex view of Industry 4.0. SAGE Open, 6 (2), 2158244016653987.

[30] Rusmann, M., M. Lorenz, P. Gerbert, M. Waldner, J. Justus, P. Engel, and Harnisch, M. (2015). Industry 4.0: The Future of Productivity and Growth in Manufacturing Industries, Boston Consulting Group.

[31] Schuster, K., L. Plumanns, K. Gros, R. Vossen, A. Richert, and Jeschke, S. (2015). Preparing for Industry 4.0-testing collaborative virtual learning environments with students and professional trainers. International Journal Advanced Corporate Learning, 8 (4), 14-20.

[32] Sanders, A., C. Elangeswaran and Wulfsberg, J. (2016). Industry 4.0 implies lean manufacturing: research activities in Industry 4.0 function as enablers for lean manufacturing. Journal of Industrial Engineering and Management, 9 (3), 811-833.

[33] Shafiq, S.I., C. Sanin, C. Toro, and Szczerbicki, E. (2015). Virtual engineering object (VEO): toward experiencebased design and manufacturing for Industry 4.0. Cybernetic Systems, 46 (1-2), 35-50.

[34] Shafiq, S.I., C. Sanin, E. Szczerbicki, and Toro, C. (2016). Virtual engineering factory: creating experience base for Industry 4.0, Cybernetics System, 47 (1-2), 32-47.

[35] Stock, T. and Seliger, G. (2016). Opportunities of sustainable manufacturing in Industry 4.0, Procedia CIRP, 40, 536-541.

[36] Thames, L. and Schaefer, D. (2016). Software-defined cloud manufacturing for Industry 4.0, Procedia CIRP, 52, $12-17$.

[37] $\mathrm{Xu}, \mathrm{L} ., \mathrm{W} . \mathrm{He}$, and Li, S. (2014). Internet of things in industries: a survey, IEEE Transactions on Industrial Informatics, 10 (4), 2233-2243.

[38] Zhai, C., Z. Zou, Q. Chen, L. Xu, L. Zheng, and Tenhunen H. (2016). Delay-aware and reliability-aware contentionfree MF-TDMA protocol for automated RFID monitoring in industrial IoT. Journal of Industrial Information Integration, 3, 8-19.

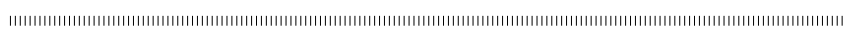
Guixiang JIN

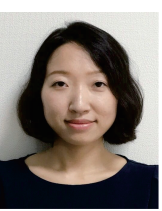

is an assistant professor in Seikei University Japan. She received Ph.D. degree from Engineering school of science and technology, Keio University. Her research interests includes safety stock, demand forecasting, logistics, and supply chain management.

\section{Akihiro Nakatsuka}

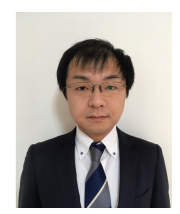

is working at dept. of supply chain management, Fuji Xerox Co., Ltd. He has experienced supply demand planning and scheduling of photocopiers and consumables. He received Ph.D. degree from Engineering school of science and technology, Keio University. He has published in the Journal of Japan Industrial Management Association (JIMA). His research interest includes supply chain management and demand forecasting, production control and logistics.

\section{Hiroaki Matsukawa}

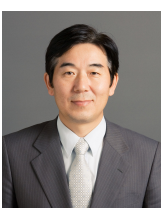

Main research interests include production \& inventory control and supply chain management (SCM). Continuous effort is dedicated to clarify principles of management on those research topics such as scheduling, manufacturing strategy, project management and other topics related to production and logistics. Quantitative methods were frequently applied for solving management problems. Closed-loop SCM and supply chain risk management are also great interesting topics.

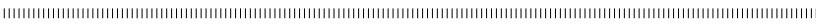

